\title{
Dietary carbohydrate's effects on lipogenesis and the relationship of lipogenesis to blood insulin and glucose concentrations
}

\author{
E. J. Parks* \\ Department of Food Science and Nutrition, University of Minnesota, Twin Cities, St. Paul, MN 55108-6099, USA
}

\begin{abstract}
The process by which dietary carbohydrate is transformed into fat in the human body is termed de novo lipogenesis. New methods for the measurement of this process in humans are available and have been used to investigate the role of the carbohydrate form (fed as a liquid or solid), the level of processing of carbohydrate in foods, and the role of lipogenesis in the control of liver triacylglycerol secretion. The present paper will discuss how research results are affected by both the physical state of the carbohydrate in the diet and by the metabolic state of individual research subjects. Of interest is the relationship between the glycemic index of a food (or indicators of a food's glycemic index) and that food's ability to stimulate lipogenesis in humans. Given the increasing prevalence of obesity worldwide, future scientific emphasis will expand methods to quantitate the lipogenic potential of specific foods and dietary patterns and investigate how the metabolic state of insulin resistance affects lipogenesis and/or contributes to obesity.
\end{abstract}

Dietary carbohydrate: Triacylglycerols: Lipogenesis: Human subjects: Feeding study

\section{Introduction}

In clinical medicine, fasting concentrations of metabolites are used for the prediction of disease risk. A physician may order a test to measure a patient's fasting blood cholesterol concentration to determine if the patient is at elevated risk for the development of coronary heart disease. Similarly, elevated fasting glucose or insulin concentration can be the first indicator that diabetes risk is high in a given patient. However, since the average person is in the fasting state only a small portion of the day (typically $6 \mathrm{~h}$ in the middle of the night), it is difficult to understand how measurement of metabolites in the fasting state can so well predict future disease risk. Most people are in the postprandial (fed) state $16-18 \mathrm{~h} /$ day and therefore, disease risk should be better predicted by a postprandial test than a fasting test. This has been found to be true for the measurement of blood triacylglycerol (TAG), which is elevated in the postprandial state in patients with documented coronary artery disease (Ginsberg et al. 1995). Indeed, research in the 1990s established that slowed postprandial metabolism of TAG after a standard high-fat meal posed a significant increase in atherogenic risk (Patsch et al. 1992). Therefore, it is reasoned, a change in dietary regimen that increases postprandial TAG might also contribute to atherogenic risk. As will be reviewed, elevations in fasting and post- prandial blood TAG concentrations occur when individuals reduce the content of fat in their diet (to $<25 \%$ of energy) and replace these calories with carbohydrate $(\mathrm{CHO})$. Such a rise in TAG as a result of increasing dietary $\mathrm{CHO}$ is known as 'CHO-induced hypertriacylglycerolemia'.

The biological process by which carbons from $\mathrm{CHO}$ (glucose, fructose, lactose, etc.) are made into fat is called de novo lipogenesis. Although de novo lipogenesis is highly active in rodents, its contribution to fatty acid flux in man has not been well defined due to a previous lack of methods to measure fat synthesis in vivo in humans. Whether an increase in de novo lipogenesis is the metabolic basis of CHO-induced hypertriacylglycerolemia is currently an extremely active area of research. The present paper will review data from human studies of lipogenesis that suggest that lipogenesis is stimulated by some, but not all high-CHO diets. The clinical implications of these diet-induced changes in blood lipids are also discussed.

\section{Increases in dietary carbohydrates lead to elevations in both fasting and postprandial lipemia}

The majority of blood TAG are carried in two types of lipoprotein particles. The first type of lipoprotein is the chylomicron, which is made in the intestine and transports

\footnotetext{
Abbreviations: BMI, body mass index; CHO, carbohydrate; TAG, triacylglyerol; VLDL, very low-density lipoprotein.

Note: For the definition of the terms inulin and oligofructose please refer to the introductory paper (p. S139) and its footnote.

* Corresponding author: Dr E. J. Parks, tel +1 612625 1785, fax +1 612625 5272, email eparks@umn.edu
} 
dietary lipid to the tissues; they are present in the blood primarily after meals. The second TAG-rich lipoprotein is the very low-density lipoprotein (VLDL), which is made in the liver and transports endogenous lipids. While most fasting TAG are carried in VLDL, elevations in postprandial blood TAG can be a result of greater TAG carried in both chylomicrons and VLDL. Numerous studies have investigated alterations in dietary $\mathrm{CHO}$, and its effects on fasting blood TAG concentrations, as reviewed in detail elsewhere (Parks \& Hellerstein, 2000). One example of data from patients with coronary artery disease is shown in Fig. 1. Thirty-four patients switched from a high-CHO (59\% of energy), low-fat $(21 \%)$ diet to one that was very high in $\mathrm{CHO}(76 \%)$, with almost no fat $(8 \%$ of energy). For the group as a whole, the average plasma TAG concentration was not different between the diets, although a highly variable response was noted among the individual subjects' data. Those with a body mass index (BMI) equal to or greater than $28 \mathrm{~kg} / \mathrm{m}^{2}$ experienced a $30 \%$ increase in TAG concentration, while those whose BMI was less than 28, experienced no change $(P<0.05$ for the interaction between treatment and BMI group). In this study, the dietary changes made were part of a comprehensive therapy designed to treat coronary atherosclerosis, and both groups of subjects lost a significant amount of body weight as a consequence of the therapy (Parks et al. 2001). These data demonstrate that certain characteristics (e.g. BMI) can make some individuals more sensitive with respect to lipid and lipoprotein changes when dietary $\mathrm{CHO}$ is increased. Such characteristics that have been identified from previous work in this field and include BMI, insulin sensitivity (Coulston et al. 1989), concentration of TAG before the dietary change is made (Parks et al. 2001), hormone replacement therapy (Kasim-Karakas et al. 2000), and genetic factors (Dreon et al. 2000).

A CHO-induced effect on fasting TAG has been established over the past four decades and it is now clear that the concentrations of both VLDL and chylomicrons are

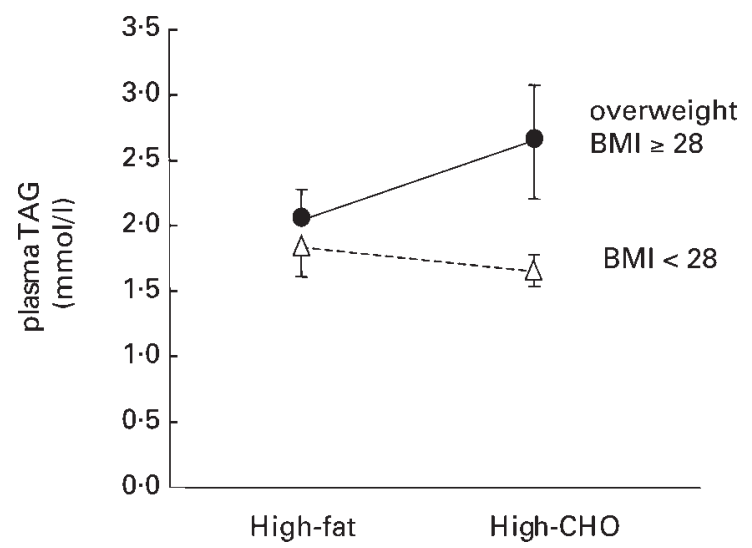

Fig. 1. Changes in fasting plasma triacylglycerol in coronary artery disease patients on diets of different macronutrient composition. Unpublished data are from Parks et al., derived from the same data set as in references (Parks et al. 1998; Parks et al. 2001). Patients were either overweight (BMI $<28 \mathrm{~kg} / \mathrm{m}^{2}, n=8$ ) or had a $\mathrm{BMI}<$ $28 \mathrm{~kg} / \mathrm{m}^{2} \quad(n=26)$. Changes in triacylglycerol concentrations between the two groups were significantly different $(P<0.05$, twoway ANOVA). Values are means (SEM). elevated in the fasting state. The effect of $\mathrm{CHO}$ feeding to increase TAG concentrations in the postprandial state is becoming more evident (Chen et al. 1995; Jeppesen et al. 1995). Current data suggest that the CHO-induced increase in fasting TAG sets the stage for higher concentrations following a meal. Over time, slower postprandial clearance of TAG-rich lipoproteins, VLDL and chylomicrons, leads to higher fasting TAG. Thus, against this higher load of TAG in the blood in the fasting state, further addition of TAG following absorption of a fatty meal leads to significantly higher postprandial TAG concentrations due to reduced clearance. However, in scientific literature, when the term 'TAG clearance' is used, it is important to delineate which of two clearance mechanisms could be effected. The first is lipase-mediated removal of lipid off of TAG-rich lipoprotein particles as they circulate in the blood. Reduced TAG clearance could occur if the amount or activity of lipoprotein lipase, the primary plasma enzyme that hydrolyzes fatty acids from TAGrich lipoproteins, could be down-regulated. The second method of TAG clearance from plasma occurs when TAG-rich lipoproteins are taken up by the liver through receptor-mediated processes (see Fig. 2). Which of these mechanisms is occurring and the relative contributions of either of them is currently unknown. It is also possible that the two TAG-rich lipoproteins could be affected differently. For instance, a down-regulation of lipoprotein lipase could increase chylomicron-TAG postprandially, while down-regulation of hepatic receptors could lead to reduced VLDL-TAG clearance.

\section{Sources of triacylglycerol-fatty acids}

The sources of fatty acids that are used for hepatic VLDLTAG synthesis are shown in Fig. 2, four potential sources are as follows: (i) fatty acids derived from the plasma nonesterified fatty acid pool, which in the fasting state, primarily originate from adipose tissue; (ii) fatty acids derived from the de novo lipogenesis pathway in the liver; (iii) fatty acids originally derived from the diet that enter the liver via chylomicron remnant clearance; and (iv) fatty acids that are stored in the liver in TAG droplets. Two candidate sources have been investigated: the non-esterified fatty acid pool (source i) and de novo fatty acids (source ii). The contribution of non-esterified fatty acids to VLDL-TAG synthesis during CHO-induced hypertriacylglycerolemia is important to measure because endogenous hypertriacylglycerolemia has been shown to result from a greater flow of non-esterified fatty acids to the liver (Kissebah et al. 1981). Endogenous hypertriacylglycerolemia is genetically controlled, manifests as elevated blood TAG and/or cholesterol concentrations on higher-fat diets, and significantly increases the risk of coronary heart disease development (Brunzell et al. 1976). If the consumption of a high-CHO diet increases VLDL-TAG production rate though a similar mechanism as that of endogenous hypertriacylglycerolemia, similar increases in coronary heart disease risk might be attributed to the two hypertriacylglycerolemias. The second source of fatty acids for VLDL-TAG synthesis is from the de novo lipogenesis pathway. De novo synthesis could be stimulated by an 


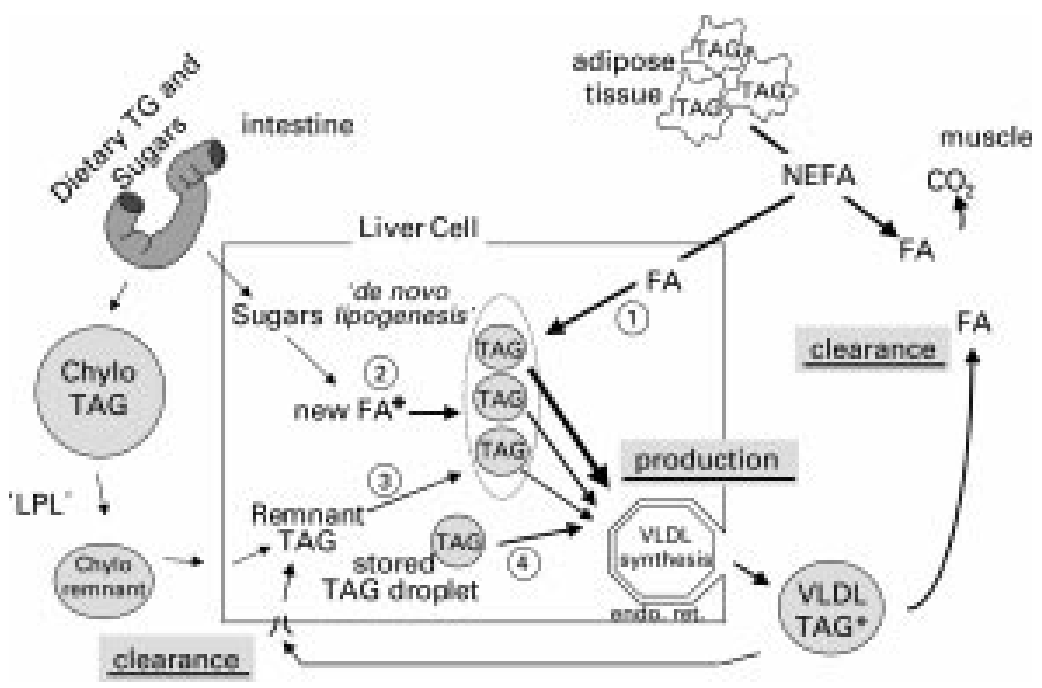

Fig. 2. Sources of fatty acids used for VLDL-TAG synthesis in the liver. The sources 1-4 are described in the text. Abbreviations: VLDL, very low-density lipoprotein; FA, fatty acid; TAG, triacylglycerol; LPL, lipoprotein lipase; NEFA, plasma non-esterified fatty acids (derived originally from adipose tissue); chylo, intestinallyderived chylomicron lipoprotein particles. The asterisk demonstrates the de novo lipogenesis pathway that can be labeled with an infusion of labeled acetate. Fatty acids derived from this source can be found in VLDL-TAG in the blood.

excess flow of glucose down glycolysis and into the hepatic acetyl-CoA pool and recently, methods to measure de novo lipogenesis in humans have been developed (Hellerstein, 1995; Hudgins et al. 1993; Jones, 1996). De novo lipogenesis data are presented in Fig. 4 and will be described later in this article. Note that de novo lipogenesis data are presented as a percentage which is interpreted as the percentage of fatty acids secreted in VLDL that are derived from this pathway. We have measured the contribution of both non-esterified fatty acids and de novo lipogenesis to VLDL-TAG synthesis in healthy men before and after 5-weeks of isoenergetic, high-CHO feeding in which the diet was rich in polysaccharides (complex $\mathrm{CHO}$ ) and high in fiber. We found no evidence that $\mathrm{CHO}$-induced hypertriacylglycerolemia resulted from elevated non-esterified fatty acid flow or de novo lipogenesis (Parks et al. 1999). The primary phenomenon of CHO-induced hypertriacylglycerolemia could be explained by a $37 \%$ reduction in TAG clearance from the blood.

A completely different result was found by Hudgins et al. who fed healthy subjects high-CHO diets rich in monoand disaccharides and consumption of these simple sugars was associated with significantly higher de novo lipogenesis (Hudgins et al. 2000). Aside from pointing out that this diet stimulated de novo lipogenesis, the reader is referred to that publication to appreciate the large variability among subjects in the magnitude of de novo lipogenesis. A particularly striking observation was that two general patterns of de novo lipogenesis were observed throughout the day: a 'constant' and a 'diurnal' pattern (Hudgins et al. 2000). For those subjects exhibiting a CHO-stimulated increase in de novo lipogenesis of the constant pattern, the percentage of VLDL-TAG fatty acids derived from the de novo pathway was high and steady throughout the 24-h of data collection. Lipogenesis was the same during all meals and even when the subjects were sleeping. For those exhibiting a diurnal pattern, the percentage of VLDL-TAG fatty acids derived from de novo synthesis was very low in the morning. Levels rose with every meal, peaked at about 22.00 hours, fell through the night, and were very low again in the morning. No clinical characteristics were found that would distinguish the subjects in the constant or diurnal groups. That is to say, the two groups had the same number of obese and lean subjects, percentages of men and women, and young and older subjects. The two groups did not differ by blood concentrations of metabolites (insulin, glucose, fatty acids, etc.) or by hormone concentrations. These observations illustrate that extremely little is known about the stimulation of fatty acid synthesis in humans. Now that accurate methods are available to measure this process in vivo, efforts should be made to assess the contribution of de novo lipogenesis to hypertriacylglycerolemia in adults with insulin resistance. Despite variability in the lipogenic potential of high-CHO diets, two important concepts have been clarified: first, high-CHO feeding results in an expansion of body $\mathrm{CHO}$ stores and more glucose oxidation in the fasting state, and second, even if $d e$ novo lipogenesis is turned on by CHO-feeding, the quantity of fat made through this process is quantitatively minor (Hellerstein et al. 1996).

\section{Lipogenesis and the effect of monosaccharides}

Since the data of Hudgins et al. showed that diets high in mono-saccharides showed higher levels of de novo lipogenesis, it might have been assumed by some that the higher the blood glucose concentration after a meal, the more likely fatty acids will be synthesized from that glucose due to an over-supply of carbon units to the liver. If 
High-fat

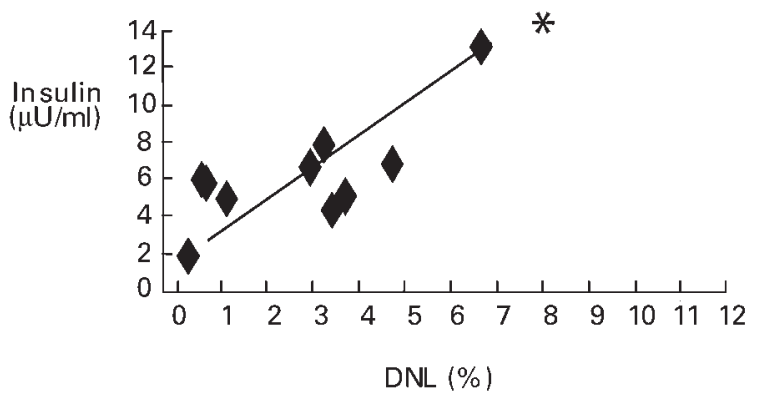

High-carbohydrate

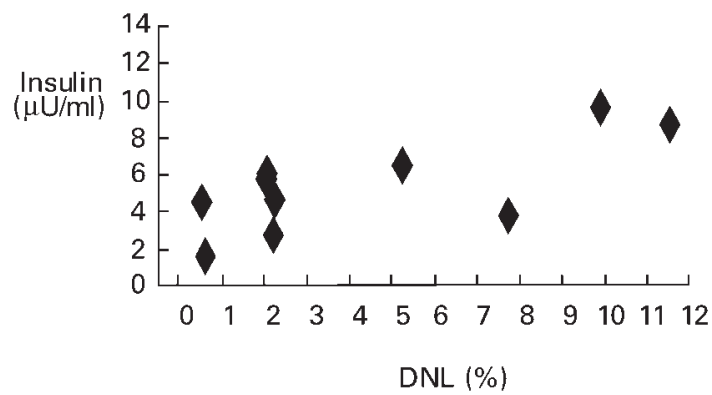

Fig. 3. The relationship between fasting insulin concentrations and de novo lipogenesis on high-fat and high-carbohydrate diets. Data are from Parks (Parks, 2002, in press).

elevated concentrations of insulin and glucose occur after higher $\mathrm{CHO}$ meals (i.e. meals with a higher glycemic index) and stimulate de novo lipogenesis, a statistical relationship might be observed between these parameters. However, no such positive relationship between fractional de novo lipogenesis and either blood insulin or glucose concentrations could be found among subjects in on high-CHO diets in data from four studies analyzed (Parks, 2002, in press). On the contrary, a positive association was found between fasting insulin concentration and de novo lipogenesis among subjects on a high-fat diet (Fig. 3). These data suggest that in healthy subjects, some other variables besides glucose and insulin are directly related to increased lipogenesis. These variables have yet to be identified and a clear relationship between the glycemic index of a meal and its ability to stimulate lipogenesis awaits further study.

\section{Effects of non-digestible carbohydrates}

The food industry is now using natural, non-digestible oligosaccharides to increase the nutritional value of foods. In healthy subjects, studies of these compounds, such as inulin and oligofructose, have shown that supplementation of the diet significantly reduced plasma lipid concentrations (Brighenti et al. 1999). Oligofructose is fermentable in the colon and the main products are short-chain fatty acids (acetic acid, propionic and butyric acid). Since the proportion of propionic and acetic acid appear to influence the synthesis of TAG and cholesterol in rodent models (as reviewed elsewhere in this Supplement), it is of interest to determine whether the same holds true in humans. One study showed a significant reduction in fasting TAG concentration by $21 \%$ after supplementation of healthy volunteers with $15 \mathrm{~g}$ oligofructose/ day. Mechanistic studies have yet to be performed to establish whether the reduction in TAG concentration is due to reduced TAG synthesis or increased clearance from the blood. It is difficult to understand how the production of acetate through gut fermentation could affect the acetylCoA pool in the liver to potentially down-regulate fatty acid synthesis. Creative strategies will be necessary to test this hypothesis in humans. Perhaps even the ends of the oligofructose molecule could be labeled to determine the extent to which the products of its fermentation ended up in the blood and in the liver. The methods are available to determine the effects of colonic fermentation of $\mathrm{CHO}$ on lipogenesis in humans.

\section{Practical implications of high-carbohydrate diets}

The difficulty experienced by clinicians whose patients lower their dietary fat and increase their $\mathrm{CHO}$ consumption is that such diets lead to both positive health benefits and changes in lipoprotein profiles that may indicate increasing disease risk (Dreon et al. 1994; Dreon et al. 1999). Health benefits result because consumption of these diets almost always results in modest weight loss (4-8 lbs over 6 weeks) and reductions in total cholesterol and LDL-cholesterol. Weight loss occurs because subjects inadvertently reduce their energy intake as a result of the increased volume of low-fat, high-fiber foods (Gambera et al. 1995; Sheppard et al. 1991). Reductions in blood cholesterol concentration can occur as a consequence of weight loss, but clearly are driven primarily by a reduction in dietary saturated fat. A reduction in dietary cholesterol content also occurs as it and saturated fat frequently occur together in foods. The paradox of CHO-induced hypertriacylglycerolemia is summarized in Figs 4 and 5. The potentially negative changes in blood lipids are evident, such as increased TAG (Fig. 3), increased VLDL-cholesterol (Fig. 5), and reduced HDL-cholesterol (Fig. 5), as are the potential benefits of this diet which include reduced total and LDL-cholesterol concentrations (Fig. 5).

These potentially negative $\mathrm{CHO}$-induced changes in lipoprotein profiles ( $\uparrow$ TAG concentrations, $\downarrow$ HDL-cholesterol, and reductions in LDL particle size in some subjects) have been identified as independent risk factors for the development of coronary heart disease (Dreon et al. 1999). It should be emphasized, however, that assignment of these changes in TAG, HDL-cholesterol, and LDL particle size as coronary heart disease risk factors resulted from comprehensive analysis of data from cross-sectional studies and long-term observational studies in subjects on higher-fat diets (Brinton et al. 1990; Brown et al. 1995; Jackson et al. 1987; Laws \& Reaven, 1992). None of these factors has been proven to increase risk when they occur solely as a result of the replacement of dietary 


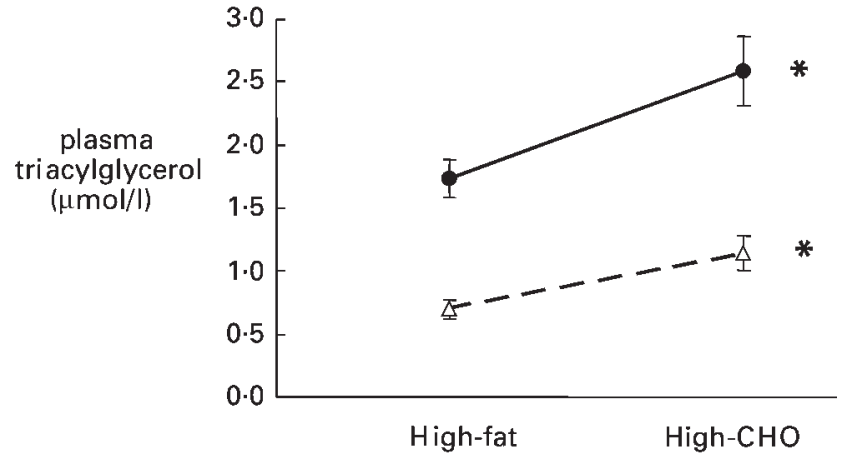

Fig. 4. Changes in fasting plasma triacylglycerol concentration in five subjects with hypertriacylglycerolemia (filled circles) and in six normolipidemics (open triangles) after switching from their habitual high-fat diet (consumed for 1 week) to a high-carbohydrate (CHO) diet (consumed for 5 weeks). The high-fat diet was 36\% fat and $50 \% \mathrm{CHO}$, while the high- $\mathrm{CHO}$ diet consisted of $15 \%$ fat and $68 \%$ $\mathrm{CHO}$. * Denotes a within-group comparison of high- $\mathrm{CHO}$ data that are significantly different compared to that from the high-fat phase. From Parks et al. (1999). Values are means (SEM).

CHO for fat. For the case of elevated TAG, risk has been identified in meta-analyses and cross-sectional studies of large populations on a wide variety of diets (Austin, 1998; Austin et al. 1988). High blood TAG concentration in individuals on typical diets confers risk, but the test of hypertriacylglycerolemia as a result of dietary change has not been tested. Put another way, no study so far has found an independent effect of TAG elevation to increase risk when LDL-cholsterol has been simultaneously
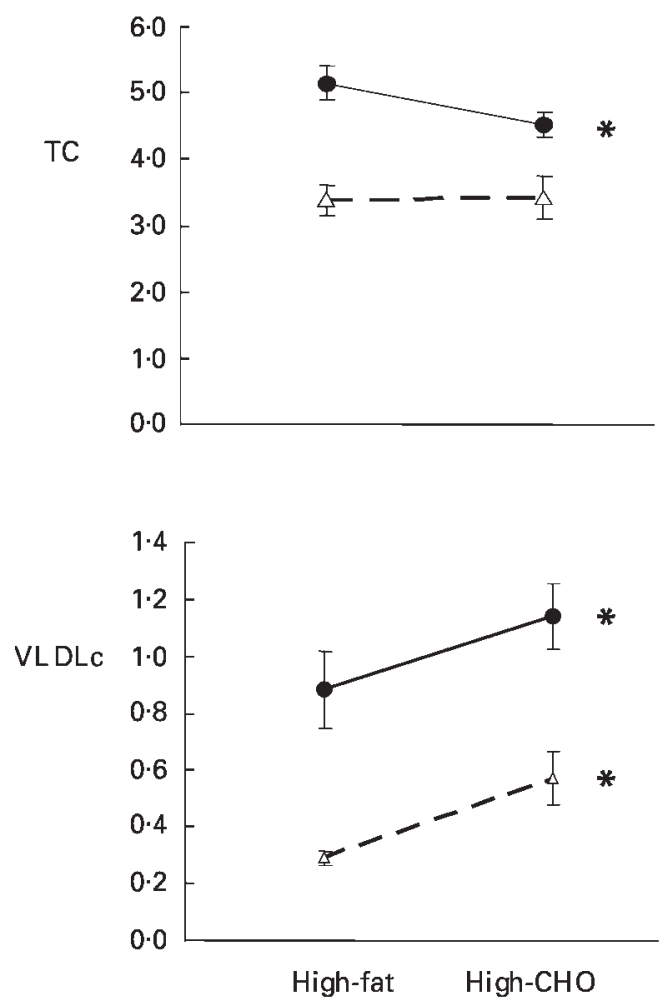

reduced. The American Heart Association has determined that although no study has been able to test this premise, very low-fat diets which raise TAG should be avoided in favor of diets higher in fat, in which the fat is composed primarily of monounsaturated fatty acids (Lichtenstein \& Van Horn, 1998).

Given a clear health benefit of reducing total and LDL cholesterol concentrations, and the suspected, but as yet unproven, evidence that the $\mathrm{CHO}$-induced hypertriacylglycerolemia increases coronary heart disease risk, clinicians are left wondering whether the sum of the CHO-induced changes provides net benefit to a patient. This question remains unanswered and the practical implications are as follows:

- Compared to high-CHO diets, higher monounsaturated-fat diets may be preferable if they do not lead to increased energy intake and weight gain, and if they do not contain higher quantities of cholesterol. Efforts should be made to make sure patients are obtaining adequate amounts of dietary vitamin $\mathrm{C}$, fiber, and carotenoids.

- Alternatively, higher-CHO diets may be well tolerated and the hypertriacylglycerolemia mitigated somewhat by the weight loss that can occur on these diets. The diets of patients particularly susceptible to CHO-induced hypertriacylglycerolemia should be monitored more often to make sure that the hypertriacylglycerolemia is not being exacerbated by over-consumption of alcohol or highly processed foods containing large amounts of sugars.
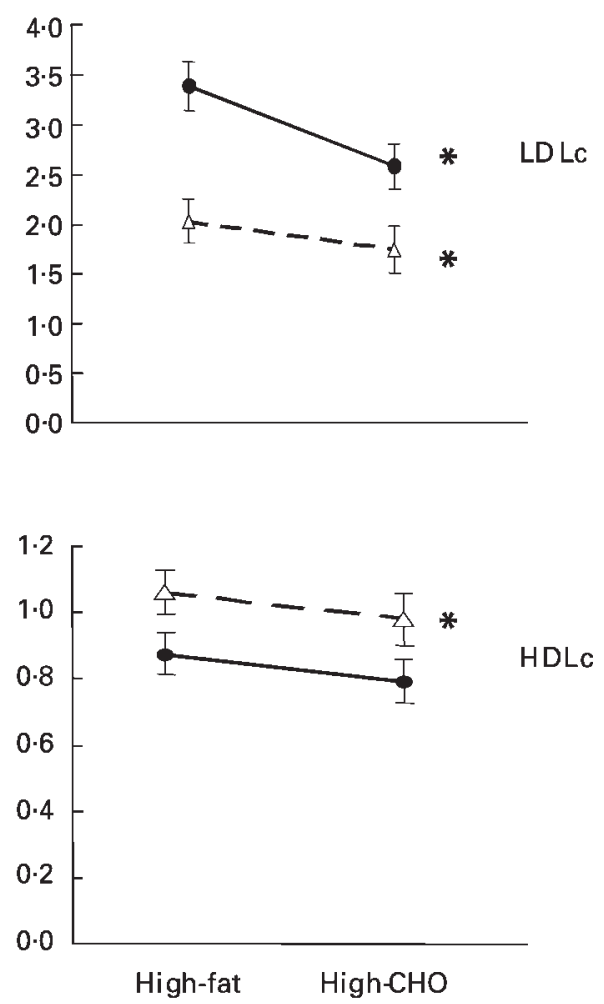

Fig. 5. Changes in plasma total cholesterol (TC) and lipoprotein cholesterol concentrations after switching from a high-fat to a high- $\mathrm{CHO}$ diet. Data are from Parks et al. (1999); see details provided with Fig. 4. All values are means (SEM) and have units of mmol/l. 


\section{Factors that effect plasma triacylglycerol concentration and future research directions}

Because plasma TAG concentration varies $10-20 \%$ from day to day, it is important to control for factors that can effect TAG status when conducting research studies designed to determine lipogenesis and fatty acid turnover. Factors that dramatically effect plasma TAG concentration include alcohol intake, hydration status, effects of a subject's background diet and effects of the meal eaten just prior to sample collection. Alcohol is clearly lipogenic (Siler et al. 1998) and alcohol consumption should be prohibited in research subjects for the $48 \mathrm{~h}$ prior to a research study. Plasma TAG can appear to be elevated if the subject is significantly dehydrated (alcohol is also dehydrating). Studies have also shown that plasma TAG can be elevated after a single high-sugar meal fed the night before blood is drawn therefore, controlling a research subject's diet before blood sampling is essential to control for these confounding variables.

\section{Summary and future research priorities}

With ready availability of accurate methods to measure lipogenesis in humans, a number of key research issues can now be investigated. Which subject characteristics (body weight, insulin concentration, etc.) are related to elevations in lipogenesis, and which hormones or genes control the diurnal pattern of fatty acid synthesis? Preliminary data suggest that the state of insulin resistance at the level of the liver may be associated with increased lipogenesis. What effect does the structure of the polysaccharide have on lipogenesis? Is the absorption rate of the $\mathrm{CHO}$ important in the lipogenic potential of the sugar? Further work is needed to clarify the mechanism of TAG reduction during fructose oligosaccharides consumption. Additional methods will be needed to quantitate de novo lipogenesis in human adipose tissue in vivo and to determine whether this amount of lipogenesis can contribute substantially to obesity. Finally, given the shift in the distribution toward elevated body weights in the population, the metabolic effects of different dietary $\mathrm{CHO}$ (some increasing lipogenesis, some potentially reducing it, etc.) will be a critical focus of future research.

\section{References}

Austin MA (1998) Plasma triglyceride as a risk factor for cardiovascular disease. Canadian Journal of Cardiology 13, 14B-17B.

Austin MA, Breslow JL, Hennekens CH, Buring JE, Willett WC \& Krauss RM (1988) Low-density lipoprotein subclass patterns and risk of myocardial infarction. Journal of the American Medical Association 260, 1917-1921.

Brighenti F, Casiraghi MC, Canzi E \& Ferrari A (1999) Effect of consumption of a ready-to-eat breakfast cereal containing inulin on the intestinal milieu and blood lipids in healthy male volunteers. European Journal of Clinical Nutrition 53, 726-733.

Brinton EA, Eisenberg S \& Breslow JL (1990) A low-fat diet decreases high-density lipoprotein (HDL) cholesterol levels by decreasing HDL apolipoprotein transport rates. Journal of Clinical Investigation 85, 144-151.

Brown BG, Zhao X-Q \& Maher VMG (1995) HDL cholesterol as a therapeutic target in coronary disease: current concepts and future directions in HDL deficiency and atherosclerosis. In Developments in Cardiovascular Medicine, Vol. 174, pp. 24-42 [G Assan, editor]. Boston, MA: Kluwer Academic Publishers.

Brunzell JD, Schrott HG, Motulsky AG \& Bierman EL (1976) Myocardial infarction in the familial forms of hypertriglyceridemia. Metabolism, Clinical and Experimental 25, 313-320.

Chen YDI, Coulston AM, Zhou MY, Hollenbeck CB \& Reaven GM (1995) Why do low-fat, high-carbohydrate diets accentuate postprandial lipemia in patients with NIDDM? Diabetes Care 18, 10-16.

Coulston AM, Hollenbeck CB, Swislocki AL \& Reaven GM (1989) Persistence of hypertriglyceridemic effects of low-fat, high-carbohydrate diets in NIDDM. Diabetes Care 12, 94-100.

Dreon DM, Fernstrom HA, Miller B \& Krauss RM (1994) Lowdensity lipoprotein subclass pattern and lipoprotein response to a reduced-fat diet in men. FASEB Journal 8, 121-126.

Dreon DM, Fernstrom HA, Williams PT \& Krauss RM (1999) A very low-fat diet is not associated with improved lipoprotein profiles in men with a predominance of large, low-density lipoproteins. American Journal of Clinical Nutrition 69 , 411-418.

Dreon DM, Fernstrom HA, Williams PT \& Krauss RM (2000) Reduced LDL particle size in children consuming a very low-fat diet is related to parental LDL-subclass patterns. American Journal of Clinical Nutrition 71, 1611-1616.

Gambera PJ, Schneeman BO \& Davis PA (1995) Use of the Food Guide Pyramid and US Dietary Guidelines to improve dietary intake and reduce cardiovascular risk in active-duty Air Force members. Journal of the American Dietetic Association 95, $1268-1273$.

Ginsberg HN, Jones J, Blaner WS, Thomas A, Karmally W, Fields L, Blood D \& Begg MD (1995) Association of postprandial triglyceride and retinyl palmitate responses with newly diagnosed exercise-induced myocardial ischemia in middleaged men and women. Arteriosclerosis Thrombosis and Vascular Biology 15, 1829-1838.

Hellerstein MK (1995) Methods for measurement of fatty acid and cholesterol metabolism. Current Opinion in Lipidology 6 , $172-181$.

Hellerstein MK, Schwarz JM \& Neese RA (1996) Regulation of hepatic de novo lipogenesis in humans. Annual Review of Nutrition 16, 523-557.

Hudgins L, Hellerstein M, Seldman C, Diakun J \& Hirsch J (1993) Increased de novo lipogenesis on a eucaloric low fat, high carbohydrate diet does not alter energy expenditure. Obesity Research 1, 92S, (abstr).

Hudgins LC, Hellerstein MK, Seidman CE, Neese RA, Tremaroli JD \& Hirsch J (2000) Relationship between carbohydrateinduced hypertriglyceridemia and fatty acid synthesis in lean and obese subjects. Journal of Lipid Research 41, 595-604.

Jackson RL, Yates MT, McNerney CA \& Kashyap ML (1987) Diet and HDL metabolism: high carbohydrate vs. high fat diets. Advances in Experimental Medicine and Biology 210 , $165-172$.

Jeppesen J, Chen YDI, Zhou MY, Wang T \& Reaven GM (1995) Effect of variations in oral fat and carbohydrate load on postprandial lipemia. American Journal of Clinical Nutrition 62 , $1201-1205$.

Jones PJH (1996) Tracing lipogenesis in humans using deuterated water. Journal of Physiology and Pharmacology 74, 755-760.

Kasim-Karakas SE, Almario RU, Mueller WM \& Peterson J (2000) Changes in plasma lipoproteins during low-fat, high- 
carbohydrate diets: effects of energy intake. American Journal of Clinical Nutrition 71, 1439-1447.

Kissebah AH, Alfarsi S \& Adams PW (1981) Integrated regulation of very low density lipoprotein triglyceride and apolipoprotein-B kinetics in man: normolipemic subjects, familial hypertriglyceridemia and familial combined hyperlipidemia. Metabolism 30, 856-868.

Laws A \& Reaven GM (1992) Evidence for an independent relationship between insulin resistance and fasting plasma HDL-cholesterol, triglyceride and insulin concentrations. Journal of Internal Medicine 231, 25-30.

Lichtenstein AH \& Van Horn L (1998) Very low fat diets. Circulation 98, 935-939.

Parks E \& Hellerstein MK (2000) Carbohydrate-induced hypertriacylglycerolemia: An historical perspective and review of biological mechanisms. American Journal of Clinical Nutrition 71, 412-433.

Parks EJ (2002) The relationship of the glycemic index to lipogenesis in humans. In Proceedings of the 6th (Millenium) Vahouny Conference, [D Kritchevsky, editor]. Kluwer/Plenum Press (in press).

Parks EJ, German JB, Davis PA, Frankel EN, Kappagoda CT, Rutledge JC, Hyson DA \& Schneeman BO (1998) Reduced susceptibility of LDL from patients participating in an intensive atherosclerosis treatment program. American Journal of Clinical Nutrition 68, 778-785.

Parks EJ, Krauss RM, Christiansen MP, Neese RA \& Hellerstein MK (1999) Effects of a low-fat, high-carbohydrate diet on VLDL-triglyceride assembly, production and clearance. Journal of Clinical Investigation 104, 1087-1096.

Parks EJ, Rutledge JC, Davis PA, Hyson DA, Schneeman BO \& Kappagoda CT (2001) Predictors of plasma triglyceride elevation in patients participating in a coronary atherosclerosis treatment program. Journal of Cardiopulmonary Rehabilitation 21, 73-79.

Patsch JR, Miesenbock G, Hopferwieser T, Muhlberger V, Knapp E, Dunn JK, Gotto AM \& Patsche W (1992) Relations of triglyceride metabolism and coronary artery disease: studies in the postprandial state. Arteriosclerosis and Thrombosis $\mathbf{1 2}$, 1336-1345.

Sheppard L, Kristal AR \& Kushi LH (1991) Weight loss in women participating in a randomized trial of low-fat diets. American Journal of Clinical Nutrition 54, 821-828.

Siler SQ, Neese RA, Parks EJ \& Hellerstein MK (1998) VLDLtriglyceride production after alcohol ingestion, studied using [2-13C1] glycerol. Journal of Lipid Research 39, 2319-2328. 